\title{
Mean-Field Forward-Backward Doubly Stochastic Differential Equations and Related Nonlocal Stochastic Partial Differential Equations
}

\author{
Qingfeng Zhu ${ }^{1,2}$ and Yufeng Shi ${ }^{2}$ \\ ${ }^{1}$ School of Mathematic and Quantitative Economics, Shandong University of Finance and Economics, Jinan 250014, China \\ ${ }^{2}$ Institute for Financial Studies and School of Mathematics, Shandong University, Jinan 250199, China \\ Correspondence should be addressed to Yufeng Shi; yfshi@sdu.edu.cn
}

Received 12 December 2013; Accepted 27 January 2014; Published 24 March 2014

Academic Editor: Litan Yan

Copyright (c) 2014 Q. Zhu and Y. Shi. This is an open access article distributed under the Creative Commons Attribution License, which permits unrestricted use, distribution, and reproduction in any medium, provided the original work is properly cited.

Mean-field forward-backward doubly stochastic differential equations (MF-FBDSDEs) are studied, which extend many important equations well studied before. Under some suitable monotonicity assumptions, the existence and uniqueness results for measurable solutions are established by means of a method of continuation. Furthermore, the probabilistic interpretation for the solutions to a class of nonlocal stochastic partial differential equations (SPDEs) combined with algebra equations is given.

\section{Introduction}

In order to provide a probabilistic interpretation for the solutions of a class of semilinear stochastic partial differential equations (SPDEs), Pardoux and Peng [1] introduced the following backward doubly stochastic differential equations (BDSDEs):

$$
\begin{aligned}
Y_{t}= & +\int_{t}^{T} f\left(s, Y_{s}, Z_{s}\right) d s+\int_{t}^{T} g\left(s, Y_{s}, Z_{s}\right) \overleftarrow{d} B_{s} \\
& -\int_{t}^{T} Z_{s} \vec{d} W_{s}, \quad 0 \leq t \leq T .
\end{aligned}
$$

Due to their important significance to SPDEs, the researches for BDSDEs have been in the ascendant (cf. [2-8] and their references).

Peng and Shi [9] introduced a type of time-symmetric forward-backward stochastic differential equations, that is, the so-called fully coupled forward-backward doubly stochastic differential equations (FBDSDEs):

$$
\begin{aligned}
y_{t}= & x+\int_{0}^{t} f\left(s, y_{s}, Y_{s}, z_{s}, Z_{s}\right) d s \\
& +\int_{0}^{t} g\left(s, y_{s}, Y_{s}, z_{s}, Z_{s}\right) \vec{d} W_{s}-\int_{0}^{t} z_{s} \overleftarrow{d} B_{s},
\end{aligned}
$$

$$
\begin{aligned}
Y_{t}= & \Phi\left(y_{T}\right)+\int_{t}^{T} F\left(s, y_{s}, Y_{s}, z_{s}, Z_{s}\right) d s \\
& +\int_{t}^{T} G\left(s, y_{s}, Y_{s}, z_{s}, Z_{s}\right) \overleftarrow{d} B_{s}+\int_{t}^{T} Z_{s} \vec{d} W_{s} .
\end{aligned}
$$

In FBDSDEs (2), the forward equation is "forward" with respect to a standard stochastic integral $\vec{d} W_{t}$, as well as "backward" with respect to a backward stochastic integral $\overleftarrow{d} B_{t}$; the coupled "backward equation" is "forward" under the backward stochastic integral $\overleftarrow{d} B_{t}$ and "backward" under the forward one. In other words, both the forward equation and the backward one are types of BDSDE (1) with different directions of stochastic integrals. Peng and Shi [9] proved the existence and uniqueness of solutions to FBDSDEs (2) with arbitrarily fixed time duration under some monotone assumptions. Zhu et al. [10] extended the results in [9] to different dimensional FBDSDEs and weakened the monotone assumptions. Zhu and Shi [11] further generalized the method of continuation by introducing the notion of bridge. FBDSDEs can provide more extensive frameworks for the probabilistic interpretation (nonlinear stochastic FeynmanKac formula) for the solutions to a class of quasilinear 
SPDEs (cf. [11]) and stochastic Hamiltonian systems arising in stochastic optimal control problems (cf. [12-14]).

McKean-Vlasov stochastic differential equation (SDE) of the form

$$
d X_{t}=b\left(X_{t}, \mu_{t}\right) d t+\vec{d} W_{t}, \quad t \in[0, T], X_{0}=x
$$

where

$$
\begin{aligned}
b\left(X_{t}, \mu_{t}\right) & =\int_{\Omega} b\left(X_{t}(\omega), X_{t}\left(\omega^{\prime}\right)\right) P\left(d \omega^{\prime}\right) \\
& =\left.\mathbb{E}\left[b\left(\xi, X_{t}\right)\right]\right|_{\xi=X_{t}},
\end{aligned}
$$

$b: \mathbb{R}^{m} \times \mathbb{R} \rightarrow \mathbb{R}$ being a (locally) bounded Borel measurable function and $\mu(t ; \cdot)$ being the probability distribution of the unknown process $X_{t}$, was suggested by Kac [15] and firstly studied by McKean [16]. So far, numerous works have been done on the SDEs of McKean-Vlasov type and their applications; see, for example, Ahmed [17], Ahmed and Ding [18], Borkar and Kumar [19], Chan [20], Crisan and Xiong [21], Kotelenez [22], Kotelenez and Kurtz [23], and so on. It is worth pointing out that (3) is a particular case of the following general version:

$$
\begin{aligned}
X_{t}= & x+\int_{0}^{t} b\left(s, X_{s}, \mathbb{E} \phi^{b}\left[s, X_{s}, \xi\right]_{\xi=X_{s}}\right) d s \\
& +\int_{0}^{t} \sigma\left(s, X_{s}, \mathbb{E} \phi^{\sigma}\left[s, X_{s}, \xi\right]_{\xi=X_{s}}\right) \vec{d} W_{s},
\end{aligned}
$$

which can be regarded as a natural generalization of classical SDEs. Mathematical mean-field approaches play a crucial role in diverse areas, such as physics, chemistry, economics, finance, and games theory; see, for example, Lasry and Lions [24], Dawson [25], and Huang et al. [26]. In a recent work of Buckdahn et al. [27], a notion of mean-field backward stochastic differential equations (MF-BSDEs)

$$
\begin{aligned}
Y_{t}= & \xi+\int_{t}^{T} \mathbb{E}^{\prime} f\left(s, \omega, \omega^{\prime}, Y_{s}(\omega), Z_{s}(\omega), Y_{s}\left(\omega^{\prime}\right), Z_{s}\left(\omega^{\prime}\right)\right) d s \\
& -\int_{t}^{T} Z_{s} \vec{d} W_{s},
\end{aligned}
$$

with $t \in[0, T]$, was introduced to investigate one special mean-field problem in a pure stochastic approach.

Mean-field backward doubly stochastic differential equations (MF-BDSDEs) of the form

$$
\begin{aligned}
Y_{t}= & +\int_{t}^{T} f\left(s, Y_{s}, Z_{s}, \Gamma^{f}\left(s, Y_{s}, Z_{s}\right)\right) d s \\
& +\int_{t}^{T} g\left(s, Y_{s}, Z_{s}, \Gamma^{g}\left(s, Y_{s}, Z_{s}\right)\right) \stackrel{\leftrightarrow}{d} B_{s}-\int_{t}^{T} Z_{s} \vec{d} W_{s}
\end{aligned}
$$

where

$$
\begin{gathered}
{\left[\Gamma^{l}\left(s, Y_{s}, Z_{s}\right)\right](\omega)} \\
\doteq \int_{\Omega} \theta^{l}\left(s, \omega, \omega^{\prime}, Y_{s}(\omega), Z_{s}(\omega), Y_{s}\left(\omega^{\prime}\right),\right. \\
\left.Z_{s}\left(\omega^{\prime}\right)\right) P\left(d \omega^{\prime}\right),
\end{gathered}
$$

with $l=f, g$, were discussed by Wang et al. [28], Du et al. [29], and Xu [30]. Under Lipschitz conditions, Du et al. [29] and Wang et al. [28], respectively, got the existence and uniqueness theorem of MF-BDSDEs. Wang et al. [28] gave one probabilistic interpretation for the solutions to a class of nonlocal SPDEs and the maximum principle of Pontryagin's type for optimal control problems of MF-BDSDEs. Under locally monotone conditions, $\mathrm{Xu}$ [30] got the existence and uniqueness theorem and comparison theorem of MFBDSDEs.

In this paper, we would like to introduce mean-field forward-backward doubly stochastic differential equations (MF-FBDSDEs) of the form

$$
\begin{aligned}
y_{t}= & x+\int_{0}^{t} \mathbb{E}^{\prime} f\left(s, \xi_{s}\right) d s+\int_{0}^{t} \mathbb{E}^{\prime} g(s, \xi) \vec{d} W_{s}-\int_{0}^{t} z_{s} \overleftarrow{d} B_{s}, \\
Y_{t}= & \mathbb{E}^{\prime} \Phi\left(y_{T}^{\prime}, y_{T}\right)-\int_{t}^{T} \mathbb{E}^{\prime} F\left(s, \xi_{s}\right) d s \\
& -\int_{t}^{T} \mathbb{E}^{\prime} G\left(s, \xi_{s}\right) \overleftarrow{d} B_{s}-\int_{t}^{T} Z_{s} \vec{d} W_{s},
\end{aligned}
$$

where

$$
\begin{aligned}
& \mathbb{E}^{\prime} l\left(s, \xi_{s}\right) \\
& =\mathbb{E}^{\prime} l\left(s, y_{s}, Y_{s}, z_{s}, Z_{s}, y_{s}^{\prime}, Y_{s}^{\prime}, z_{s}^{\prime}, Z_{s}^{\prime}\right) \\
& =\int_{\Omega} l\left(s, \omega, \omega^{\prime}, y_{s}(\omega), Y_{s}(\omega), z_{s}(\omega), Z_{s}(\omega), y_{s}\left(\omega^{\prime}\right),\right. \\
& \left.Y_{s}\left(\omega^{\prime}\right), z_{s}\left(\omega^{\prime}\right), Z_{s}\left(\omega^{\prime}\right)\right) P\left(d \omega^{\prime}\right), \\
& \quad l=f, g, F, G, \\
& \mathbb{E}^{\prime} \Phi\left(y_{T}, y_{T}^{\prime}\right)=\int_{\Omega} \Phi\left(\omega, \omega^{\prime}, y_{T}(\omega), y_{T}\left(\omega^{\prime}\right)\right) P\left(d \omega^{\prime}\right) .
\end{aligned}
$$

Following the basic ideas in [1], we firstly discuss the existence and uniqueness of solutions for MF-FBDSDE (9), which obviously extends the results in [9], Wang et al. [28], Du et al. [29], and $\mathrm{Xu}$ [30]. It is worth pointing out that MF-FBDSDE is not just a natural generalization of FBDSDE and MF-BDSDE from the view of mathematics. Our study on them also is motivated by the probabilistic interpretation for the solutions to some kind of nonlocal SPDEs.

As is well known to us, the research on SPDEs has increasingly been a popular issue in recent years. As one kind of them, SPDEs of the McKean-Vlasov type were discussed in [23]. In fact, such equations were obtained as continuum 
limit from empirical distributions of a large number of SDEs, coupled with mean-field interaction. We also refer the readers to $[21,22]$ for more details along this. On the other hand, we would also like to mention the work of Buckdahn et al. [31] who studied one kind of nonlocal deterministic PDEs. In virtue of the "backward semigroup" method, they obtained the existence and uniqueness of viscosity solution for nonlocal PDEs via MF-BSDE (6) in a Markovian framework and McKean-Vlasov forward equations. Furthermore, $\mathrm{Wu}$ and $\mathrm{Yu}$ $[32,33]$ and Li and Wei [34] investigated PDE combined with algebra equations. Motivated by the above three cases, in this paper we will give some discussions on one kind of nonlocal SPDEs. A probabilistic interpretation for the solutions to such kind of SPDEs is derived by virtue of a connection between them and fully coupled FBDSDEs of mean-field type, which extends the results in [11] to the mean-field case and extends the results in $[32,33]$ to stochastic case.

The paper is organized as follows. In Section 2, we will present some preliminary notations needed in the whole paper. In Section 3, we consider the existence and uniqueness of solutions for MF-FBDSDE. In Section 4, we give the probabilistic interpretations for the solutions to a class of nonlocal SPDEs by means of MF-FBDSDEs.

\section{Setting of the Problem}

Let $(\Omega, \mathscr{F}, P)$ be a complete probability space on which are defined two mutually independent Brownian motions $\left\{W_{t}\right\}_{t \geq 0}$ and $\left\{B_{t}\right\}_{t \geq 0}$, with value, respectively, in $\mathbb{R}^{d}$ and $\mathbb{R}^{l}$. We denote

$$
\mathscr{F}_{t} \doteq \mathscr{F}_{t}^{W} \vee \mathscr{F}_{t, T}^{B}, \quad \forall t \in[0, T],
$$

where $\mathcal{N}$ is the class of $P$-null sets of $\mathscr{F}$ and

$$
\begin{aligned}
& \mathscr{F}_{t}^{W} \doteq \sigma\left\{W_{r} ; 0 \leq r \leq t\right\} \vee \mathcal{N}, \\
& \mathscr{F}_{t, T}^{B} \doteq \sigma\left\{B_{T}-B_{r} ; t \leq r \leq T\right\} \vee \mathcal{N} .
\end{aligned}
$$

In this case, the collection $\left\{\mathscr{F}_{t}, t \in[0, T]\right\}$ is neither increasing nor decreasing, while $\left\{\mathscr{F}_{t}^{W}, t \in[0, T]\right\}$ is an increasing filtration and $\left\{\mathscr{F}_{t, T}^{B}, t \in[0, T]\right\}$ is a decreasing filtration.

Let $\left(\Omega^{2}, \mathscr{F}^{2}, P^{2}\right)=(\Omega \times \Omega, \mathscr{F} \otimes \mathscr{F}, P \otimes P)$ be the completion of the product probability space of the above $(\Omega, \mathscr{F}, P)$ with itself, where we define $\mathscr{F}_{t}^{2}=\mathscr{F}_{t} \otimes \mathscr{F}_{t}$ with $t \in[0, T]$ and $\mathscr{F}_{t} \otimes \mathscr{F}_{t}$ being the completion of $\mathscr{F}_{t} \times \mathscr{F}_{t}$. It is worth noting that any random variable $\xi=\xi(\omega)$ defined on $\Omega$ can be extended naturally to $\Omega^{2}$ as $\xi^{\prime}\left(\omega, \omega^{\prime}\right)=\xi(\omega)$ with $\left(\omega, \omega^{\prime}\right) \epsilon$ $\Omega^{2}$. For $H=\mathbb{R}^{n}$, and so forth, let $L^{1}\left(\Omega^{2}, \mathscr{F}^{2}, P^{2} ; H\right)$ be the set of random variables $\xi: \Omega^{2} \rightarrow H$ which is $\mathscr{F}^{2}$-measurable such that $\mathbb{E}^{2}|\xi| \equiv \int_{\Omega^{2}}\left|\xi\left(\omega^{\prime}, \omega\right)\right| P\left(d \omega^{\prime}\right) P(d \omega)<\infty$. For any $\eta \in L^{1}\left(\Omega^{2}, \mathscr{F}^{2}, P^{2} ; H\right)$, we denote

$$
\mathbb{E}^{\prime} \eta(\omega, \cdot) \doteq \int_{\Omega} \eta\left(\omega, \omega^{\prime}\right) P\left(d \omega^{\prime}\right)
$$

Particularly, for example, if $\eta_{1}\left(\omega, \omega^{\prime}\right)=\eta_{1}\left(\omega^{\prime}\right)$, then

$$
\mathbb{E}^{\prime} \eta_{1}=\int_{\Omega} \eta_{1}\left(\omega^{\prime}\right) P\left(d \omega^{\prime}\right)=\mathbb{E} \eta_{1} .
$$

We would like to introduce some spaces of functions required in the sequel:

$$
\begin{aligned}
& S^{2}\left([0, T] ; \mathbb{R}^{n}\right) \\
& =\left\{\varphi:[0, T] \times \Omega \longrightarrow \mathbb{R}^{n} \mid \varphi_{t} \text { is } \mathscr{F}_{t}\right. \text {-measurable } \\
& \left.\quad \text { process such that } \mathbb{E}\left(\sup _{0 \leq t \leq T}\left|\varphi_{t}\right|^{2}\right)<\infty\right\}, \\
& M^{2}\left(0, T ; \mathbb{R}^{n}\right) \\
& =\left\{\varphi:[0, T] \times \Omega \longrightarrow \mathbb{R}^{n} \mid \varphi_{t} \text { is } \mathscr{F}_{t}\right. \text {-measurable process } \\
& \left.\quad \text { such that } \mathbb{E} \int_{0}^{T}\left|\varphi_{t}\right|^{2} d t<\infty\right\}, \\
& L^{2}\left(\Omega, \mathscr{F}_{T}, P ; \mathbb{R}^{n}\right) \\
& =\left\{\xi:[0, T] \times \Omega \longrightarrow \mathbb{R}^{n} \mid \xi \text { is } \mathscr{F}_{T}\right. \text {-measurable random } \\
& \left.\quad \text { variable such that } \mathbb{E}^{n}|\xi|^{2}<\infty\right\} .
\end{aligned}
$$

We will give notations as follows:

$$
\begin{gathered}
U=\left(\begin{array}{c}
y \\
Y \\
z \\
Z
\end{array}\right), \quad U^{\prime}=\left(\begin{array}{c}
y^{\prime} \\
Y^{\prime} \\
z^{\prime} \\
Z^{\prime}
\end{array}\right), \\
\xi=\left(\begin{array}{c}
U \\
U^{\prime}
\end{array}\right), \quad A(t, \xi)=\left(\begin{array}{c}
-F \\
f \\
-G \\
g
\end{array}\right)(t, \xi) .
\end{gathered}
$$

Let $\mathbb{R}^{n}$ be the $n$-dimensional Euclidean space with the usual Euclidean norm $|\cdot|$ and the usual Euclidean inner product $\langle\cdot, \cdot\rangle$. The notation $T$ appearing in the superscripts denotes the transpose of a matrix. Also, let $\mathbb{R}^{n \times l}$ be the Hilbert space that consists of all $n \times l$-matrices with the inner product $\langle A, B\rangle=\operatorname{tr}\left\{\mathrm{AB}^{\mathrm{T}}\right\}, \forall A, B \in \mathbb{R}^{n \times d}$. Thus, the norm $|A|$ of $A \in \mathbb{R}^{n \times d}$ is given by $|A|=\sqrt{\operatorname{tr}\left\{\mathrm{AA}^{\mathrm{T}}\right\}}$. Let $S^{n}$ be the set of all $n \times n$ symmetric matrices. All the equalities and inequalities mentioned in this paper are in the sense of $d t \times d P$ almost surely on $[0, T] \times \Omega$.

Consider the following MF-FBDSDEs:

$$
\begin{aligned}
y_{t}= & x+\int_{0}^{t} \mathbb{E}^{\prime} f\left(s, \xi_{s}\right) d s+\int_{0}^{t} \mathbb{E}^{\prime} g\left(s, \xi_{s}\right) \vec{d} W_{s}-\int_{0}^{t} z_{s} \overleftarrow{d} B_{s}, \\
Y_{t}= & \mathbb{E}^{\prime} \Phi\left(y_{T}^{\prime}, y_{T}\right)-\int_{t}^{T} \mathbb{E}^{\prime} F\left(s, \xi_{s}\right) d s \\
& -\int_{t}^{T} \mathbb{E}^{\prime} G\left(s, \xi_{s}\right) \overleftarrow{d} B_{s}-\int_{t}^{T} Z_{s} \vec{d} W_{s},
\end{aligned}
$$


where

$$
\begin{aligned}
\xi_{s}= & \left(y_{s}, Y_{s}, z_{s}, Z_{s}, y_{s}^{\prime}, Y_{s}^{\prime}, z_{s}^{\prime}, Z_{s}^{\prime}\right), \\
F: \Omega & \times[0, T] \times \mathbb{R}^{n} \times \mathbb{R}^{n} \times \mathbb{R}^{n \times l} \times \mathbb{R}^{n \times d} \\
& \times \mathbb{R}^{n} \times \mathbb{R}^{n} \times \mathbb{R}^{n \times l} \times \mathbb{R}^{n \times d} \longrightarrow \mathbb{R}^{n}, \\
f: \Omega & \times[0, T] \times \mathbb{R}^{n} \times \mathbb{R}^{n} \times \mathbb{R}^{n \times l} \times \mathbb{R}^{n \times d} \\
& \times \mathbb{R}^{n} \times \mathbb{R}^{n} \times \mathbb{R}^{n \times l} \times \mathbb{R}^{n \times d} \longrightarrow \mathbb{R}^{n}, \\
G: \Omega & \times[0, T] \times \mathbb{R}^{n} \times \mathbb{R}^{n} \times \mathbb{R}^{n \times l} \times \mathbb{R}^{n \times d} \\
& \times \mathbb{R}^{n} \times \mathbb{R}^{n} \times \mathbb{R}^{n \times l} \times \mathbb{R}^{n \times d} \longrightarrow \mathbb{R}^{n \times l}, \\
g: \Omega & \times[0, T] \times \mathbb{R}^{n} \times \mathbb{R}^{n} \times \mathbb{R}^{n \times l} \times \mathbb{R}^{n \times d} \\
& \times \mathbb{R}^{n} \times \mathbb{R}^{n} \times \mathbb{R}^{n \times l} \times \mathbb{R}^{n \times d} \longrightarrow \mathbb{R}^{n \times d}, \\
\Phi: & \Omega \times \mathbb{R}^{n} \times \mathbb{R}^{n} \longrightarrow \mathbb{R}^{n} .
\end{aligned}
$$

Note that the integral with respect to $\left\{B_{t}\right\}$ is a "backward Itô integral," in which the integrand takes values at the right end points of the subintervals in the Riemann type sum, and the integral with respect to $\left\{W_{t}\right\}$ is a standard forward Itô integral. These two types of integrals are particular cases of the ItôSokorohod integral (for details refer to [35]).

Definition 1. A quadruple of $\mathscr{F}_{t}$-measurable processes $(y, Y, z, Z) \in M^{2}\left(0, T ; \mathbb{R}^{n+n+n \times l+n \times d}\right)$ is called a solution of MF-FBDSDEs (17), if (17) is satisfied.

One assumes the following.

(H1) For each $\xi \in \mathbb{R}^{n+n+n \times l+n \times d+n+n+n \times l+n \times d}, A(\cdot, \xi)$ is an $\mathscr{F}_{t}$-measurable process defined on $[0, T]$ with $A(\cdot, 0) \in$ $M^{2}\left(0, T ; \mathbb{R}^{n+n+n \times l+n \times d+n+n+n \times l+n \times d}\right)$.

(H2) $A(t, \xi)$ and $\Phi(y)$ satisfy the Lipschitz conditions: there exist constants $k>0$ and $0<\lambda<1 / 2$ such that

$$
\begin{aligned}
& |f(t, \xi)-f(t, \bar{\xi})|^{2} \leq k\left(|\hat{y}|^{2}+|\hat{Y}|^{2}+|\hat{z}|^{2}+|\widehat{Z}|^{2}\right. \\
& \left.+\left|\widehat{y}^{\prime}\right|^{2}+\left|\widehat{Y}^{\prime}\right|^{2}+\left|\widehat{z}^{\prime}\right|^{2}+\left|\widehat{Z}^{\prime}\right|^{2}\right), \\
& |F(t, \xi)-F(t, \bar{\xi})|^{2} \leq k\left(|\hat{y}|^{2}+|\widehat{Y}|^{2}+|\widehat{z}|^{2}+|\widehat{Z}|^{2}\right. \\
& \left.+\left|\widehat{y}^{\prime}\right|^{2}+\left|\widehat{Y}^{\prime}\right|^{2}+\left|\widehat{z}^{\prime}\right|^{2}+\left|\widehat{Z}^{\prime}\right|^{2}\right), \\
& |g(t, \xi)-g(t, \bar{\xi})|^{2} \leq k\left(|\widehat{y}|^{2}+|\widehat{Y}|^{2}+|\widehat{Z}|^{2}+\left|\hat{y}^{\prime}\right|^{2}\right. \\
& \left.+\left|\widehat{Y}^{\prime}\right|^{2}+\left|\widehat{Z}^{\prime}\right|^{2}\right)+\lambda\left(|\bar{z}|^{2}+\left|\hat{z}^{\prime}\right|^{2}\right), \\
& |G(t, \xi)-G(t, \bar{\xi})|^{2} \leq k\left(|\hat{y}|^{2}+|\widehat{Y}|^{2}+|\widehat{z}|^{2}+\left|\hat{y}^{\prime}\right|^{2}\right. \\
& \left.+\left|\widehat{Y}^{\prime}\right|^{2}+\left|\widehat{z}^{\prime}\right|^{2}\right)+\lambda\left(|\widehat{Z}|^{2}+\left|\widehat{Z}^{\prime}\right|^{2}\right),
\end{aligned}
$$

$\forall \xi, \quad \bar{\xi} \in \mathbb{R}^{n+n+n \times l+n \times d+n+n+n \times l+n \times d}, \quad \forall t \in[0, T]$,

$$
\begin{aligned}
\hat{y}=y-\bar{y}, \quad \widehat{Y}=Y-\bar{Y}, \quad \widehat{z}=z-\bar{z}, \quad \widehat{Z}=Z-\bar{Z}, \\
\hat{y}^{\prime}=y^{\prime}-\bar{y}^{\prime}, \quad \widehat{Y}^{\prime}=Y^{\prime}-\bar{Y}^{\prime}, \\
\widehat{z}^{\prime}=z^{\prime}-\bar{z}^{\prime}, \quad \widehat{Z}^{\prime}=Z^{\prime}-\bar{Z}^{\prime}, \\
\left|\Phi\left(y, y^{\prime}\right)-\Phi\left(\bar{y}, \bar{y}^{\prime}\right)\right| \leq k|y-\bar{y}|+k\left|y^{\prime}-\bar{y}^{\prime}\right|, \\
\forall y, \quad \bar{y} \in \mathbb{R}^{n} .
\end{aligned}
$$

The following monotonic conditions, introduced in [9], are main assumptions in this paper.

(H3)

$$
\begin{array}{r}
\mathbb{E}^{\prime}\langle A(t, \xi)-A(t, \bar{\xi}), U-\bar{U}\rangle \leq-\mu|U-\bar{U}|^{2}, \\
\forall U=(y, Y, z, Z)^{T}, \quad \bar{U}=(\bar{y}, \bar{Y}, \bar{z}, \bar{Z})^{T}, \\
U^{\prime}=\left(y^{\prime}, Y^{\prime}, z^{\prime}, Z^{\prime}\right)^{T}, \\
\bar{U}^{\prime}=\left(\bar{y}^{\prime}, \bar{Y}^{\prime}, \bar{z}^{\prime}, \bar{Z}^{\prime}\right)^{T} \in \mathbb{R}^{n} \times \mathbb{R}^{n} \times \mathbb{R}^{n \times l} \times \mathbb{R}^{n \times d}, \\
\forall t \in[0, T], \\
\mathbb{E}^{\prime}\left\langle\Phi\left(y, y^{\prime}\right)-\Phi\left(\bar{y}, \bar{y}^{\prime}\right), y-\bar{y}\right\rangle \geq \beta|y-\bar{y}|^{2}, \\
\forall y, \quad \bar{y} \in \mathbb{R}^{n},
\end{array}
$$

where $\mu$ and $\beta$ are positive constants.

\section{The Unique Solvability of MF-FBDSDEs}

In order to prove the existence and uniqueness result for (17) under $(\mathrm{H} 1)-(\mathrm{H} 3)$, we need the following lemma. The lemma involves a priori estimates of solutions of the following family of MF-FBDSDEs parametrized by $\alpha \in[0,1]$ :

$$
\begin{aligned}
d y_{t}= & {\left[\alpha \mathbb{E}^{\prime} f\left(t, \xi_{t}\right)+f_{0}(t)\right] d t-z_{t} \overleftarrow{d} B_{t} } \\
& +\left[\alpha \mathbb{E}^{\prime} g\left(t, \xi_{t}\right)+g_{0}(t)\right] \vec{d} W_{t}, \\
d Y_{t}= & {\left[\alpha \mathbb{E}^{\prime} F\left(t, \xi_{t}\right)-(1-\alpha) \mu y_{t}+F_{0}(t)\right] d t+Z_{t} \vec{d} W_{t} } \\
& +\left[\alpha \mathbb{E}^{\prime} G\left(t, \xi_{t}\right)-(1-\alpha) \mu z_{t}+G_{0}(t)\right] \overleftarrow{d} B_{t}, \\
y_{0}= & x, \quad Y_{T}=\alpha \mathbb{E}^{\prime} \Phi\left(y_{T}^{\prime}, y_{T}\right)+(1-\alpha) y_{T}+\varphi,
\end{aligned}
$$

where $\xi=\left(y, Y, z, Z, y^{\prime}, Y^{\prime}, z^{\prime}, Z^{\prime}\right)$, and $\left(F_{0}, f_{0}, G_{0}, g_{0}\right) \in$ $M^{2}\left(0, T ; R^{n+n+n \times l+n \times d}\right)$, and $\varphi \in L^{2}\left(\Omega, \mathscr{F}_{T}, P ; R^{n}\right)$ are arbitrarily given vector-valued random variables.

When $\alpha=1$, the existence of the solution of (21) implies clearly that of (17). Due to the existence and uniqueness of MF-BDSDE [28], when $\alpha=0,(21)$ is uniquely solvable. The following a priori lemma is a key step in the proof of the method of continuation. It shows that, for a fixed 
$\alpha=\alpha_{0} \in[0,1)$, if (21) is uniquely solvable, then it is also uniquely solvable for any $\alpha \in\left[\alpha_{0}, \alpha_{0}+\delta_{0}\right]$, for some positive constant $\delta_{0}$ independent of $\alpha_{0}$.

Lemma 2. Under assumptions (H1)-(H3), there exists a positive constant $\delta_{0}$ such that if, a priori, for some $\alpha_{0} \in[0,1)$ and for each $x \in \mathbb{R}^{n}, \varphi \in L^{2}\left(\Omega, \mathscr{F}_{T}, P ; \mathbb{R}^{n}\right),\left(F_{0}, f_{0}, G_{0}, g_{0}\right) \in$ $M^{2}\left(0, T ; \mathbb{R}^{n+n+n \times l+n \times d}\right)$, (21) has a unique solution, then, for each $\alpha \in\left[\alpha_{0}, \alpha_{0}+\delta_{0}\right]$ and $x \in \mathbb{R}^{n}, \varphi \in L^{2}\left(\Omega, \mathscr{F}_{T}, P ; \mathbb{R}^{n}\right)$, $\left(F_{0}, f_{0}, G_{0}, g_{0}\right) \in M^{2}\left(0, T ; \mathbb{R}^{n+n+n \times l+n \times d}\right)$, (21) also has a unique solution in $M^{2}\left(0, T ; \mathbb{R}^{n+n+n \times l+n \times d}\right)$.

Proof. Let

$$
\begin{aligned}
& U=(y, Y, z, Z), \quad \widetilde{U}=(\tilde{y}, \tilde{Y}, \widetilde{z}, \widetilde{Z}), \\
& \bar{U}=(\bar{y}, \bar{Y}, \bar{z}, \bar{Z}), \quad \overline{\widetilde{U}}=(\overline{\widetilde{y}}, \overline{\widetilde{Y}}, \overline{\widetilde{z}}, \overline{\widetilde{Z}}), \\
& \xi=\left(y^{\prime}, Y^{\prime}, z^{\prime}, Z^{\prime}, y, Y, z, Z\right), \\
& \widetilde{\xi}=\left(\widetilde{y}^{\prime}, \widetilde{Y}^{\prime}, \widetilde{z}^{\prime}, \widetilde{Z}^{\prime}, \tilde{y}, \tilde{Y}, \widetilde{z}, \widetilde{Z}\right), \\
& \bar{\xi}=\left(\bar{y}^{\prime}, \bar{Y}^{\prime}, \bar{z}^{\prime}, \bar{Z}^{\prime}, \bar{y}, \bar{Y}, \bar{z}, \bar{Z}\right), \\
& \overline{\widetilde{\xi}}=\left(\overline{\bar{y}}^{\prime}, \overline{\widetilde{Y}}^{\prime}, \overline{\bar{z}}^{\prime}, \overline{\widetilde{Z}}^{\prime}, \overline{\tilde{y}}, \overline{\widetilde{Y}}, \overline{\widetilde{z}}, \overline{\widetilde{Z}}\right), \\
& \widehat{\xi}=\xi-\widetilde{\xi}, \quad \widehat{\bar{\xi}}=\bar{\xi}-\widetilde{\bar{\xi}}, \\
& \widehat{U}=(\widehat{y}, \widehat{Y}, \widehat{z}, \widehat{Z})=(y-\tilde{y}, Y-\tilde{Y}, z-\widetilde{z}, Z-\widetilde{Z}), \\
& \widehat{\bar{U}}=(\hat{\bar{y}}, \widehat{\bar{Y}}, \widehat{\bar{z}}, \widehat{\bar{Z}})=(\bar{y}-\overline{\widetilde{y}}, \bar{Y}-\overline{\widetilde{Y}}, \bar{z}-\overline{\widetilde{z}}, \bar{Z}-\overline{\widetilde{Z}}) .
\end{aligned}
$$

Since for any $x \in \mathbb{R}^{n}, \quad\left(F_{0}, f_{0}, G_{0}, g_{0}\right) \quad \epsilon$ $M^{2}\left(0, T ; \mathbb{R}^{n+n+n \times l+n \times d}\right), \varphi \in L^{2}\left(\Omega, \mathscr{F}_{T}, P ; \mathbb{R}^{n}\right)$, there exists a unique solution to (21) for $\alpha=\alpha_{0}$, thus, for each $\bar{U}=(\bar{y}, \bar{Y}, \bar{z}, \bar{Z}) \in M^{2}\left(0, T ; \mathbb{R}^{n+n+n \times l+n \times d}\right)$, there exists a unique quadruple $U=(y, Y, z, Z) \in M^{2}\left(0, T ; \mathbb{R}^{n+n+n \times l+n \times d}\right)$ satisfying the following equations:

$$
\begin{aligned}
d y_{t}= & {\left[\alpha_{0} \mathbb{E}^{\prime} f\left(t, \xi_{t}\right)+\delta \mathbb{E}^{\prime} f\left(t, \bar{\xi}_{t}\right)+f_{0}(t)\right] d t-z_{t} \overleftarrow{d} B_{t} } \\
& +\left[\alpha_{0} \mathbb{E}^{\prime} g\left(t, \xi_{t}\right)+\delta \mathbb{E}^{\prime} g\left(t, \bar{\xi}_{t}\right)+g_{0}(t)\right] \vec{d} W_{t} \\
d Y_{t}= & {\left[\alpha_{0} \mathbb{E}^{\prime} F\left(t, \xi_{t}\right)-\left(1-\alpha_{0}\right) \mu y_{t}\right.} \\
& \left.+\delta\left(\mathbb{E}^{\prime} F\left(t, \bar{\xi}_{t}\right)+\mu \bar{y}_{t}\right)+F_{0}(t)\right] d t+Z_{t} \vec{d} W_{t} \\
+ & {\left[\alpha_{0} \mathbb{E}^{\prime} G\left(t, \xi_{t}\right)-\left(1-\alpha_{0}\right) \mu z_{t}\right.} \\
& \left.+\delta\left(\mathbb{E}^{\prime} G\left(t, \bar{\xi}_{t}\right)+\mu \bar{z}_{t}\right)+G_{0}(t)\right] \overleftarrow{d} B_{t},
\end{aligned}
$$

$$
\begin{aligned}
y_{0}= & x, \\
Y_{T}= & \alpha_{0} \mathbb{E}^{\prime} \Phi\left(y_{T}^{\prime}, y_{T}\right)+\left(1-\alpha_{0}\right) y_{T} \\
& +\delta\left(\mathbb{E}^{\prime} \Phi\left(\bar{y}_{T}^{\prime}, \bar{y}_{T}\right)-\bar{y}_{T}\right)+\varphi,
\end{aligned}
$$

where $\delta$ is a positive number independent of $\alpha_{0}$ and less than 1. We will prove that the mapping defined by

$$
\begin{aligned}
U & =I_{\alpha_{0}+\delta}(\bar{U}): M^{2}\left(0, T ; \mathbb{R}^{n+n+n \times l+n \times d}\right) \\
& \longrightarrow M^{2}\left(0, T ; \mathbb{R}^{n+n+n \times l+n \times d}\right)
\end{aligned}
$$

is contractive for a small enough $\delta$. Let $\overline{\widetilde{U}}=(\overline{\widetilde{y}}, \overline{\tilde{Y}}, \overline{\widetilde{z}}, \overline{\widetilde{Z}}) \epsilon$ $M^{2}\left(0, T ; \mathbb{R}^{n+n+n \times l+n \times d}\right)$ and $\widetilde{U}=(\tilde{y}, \tilde{Y}, \widetilde{z}, \widetilde{Z})=I_{\alpha_{0}+\delta}(\overline{\widetilde{U}})$.

Applying Itô's formula to $\langle\widehat{y}, \widehat{Y}\rangle$ on $[0, T]$, it follows that

$$
\begin{aligned}
& \mathbb{E}\left\langle\hat{y}_{T}, \alpha_{0} \widehat{\Phi}\left(y_{T}\right)+\left(1-\alpha_{0}\right) \widehat{y}_{T}\right\rangle \\
& -\mathbb{E} \int_{0}^{T} \mathbb{E}^{\prime}\left\langle\alpha_{0}\left(A\left(t, \xi_{t}\right)-A\left(t, \widetilde{\xi}_{t}\right)\right), \widehat{U}_{t}\right\rangle d t \\
& +\left(1-\alpha_{0}\right) \mu \mathbb{E} \int_{0}^{T}\left(\left|\widehat{y}_{t}\right|^{2}+\left|\widehat{z}_{t}\right|^{2}\right) d t \\
& =\mathbb{E}\left\langle\widehat{y}_{T}, \delta \widehat{\bar{y}}_{T}\right\rangle-\mathbb{E}\left\langle\widehat{y}_{T}, \delta \widehat{\Phi}\left(\bar{y}_{T}\right)\right\rangle \\
& +\delta \mathbb{E} \int_{0}^{T}\left(\left\langle\widehat{Y}_{t}, \widehat{f}\left(t, \bar{\xi}_{t}\right)\right\rangle+\left\langle\widehat{y}_{t}, \widehat{F}\left(t, \bar{\xi}_{t}\right)\right\rangle\right. \\
& \left.\quad+\left\langle\widehat{Z}_{t}, \widehat{g}\left(t, \bar{\xi}_{t}\right)\right\rangle+\left\langle\widehat{z}_{t}, \widehat{G}\left(t, \bar{\xi}_{t}\right)\right\rangle\right) d t \\
& \quad+\delta \mu \mathbb{E} \int_{0}^{T}\left(\left\langle\widehat{y}_{t}, \widehat{\bar{y}}_{t}\right\rangle+\left\langle\widehat{z}_{t}, \widehat{\bar{z}}_{t}\right\rangle\right) d t,
\end{aligned}
$$

where

$$
\begin{aligned}
& \widehat{f}\left(t, \bar{\xi}_{t}\right)=\mathbb{E}^{\prime} f\left(t, \bar{\xi}_{t}\right)-\mathbb{E}^{\prime} f\left(t, \overline{\widetilde{\xi}}_{t}\right), \\
& \widehat{g}\left(t, \bar{\xi}_{t}\right)=\mathbb{E}^{\prime} g\left(t, \bar{\xi}_{t}\right)-\mathbb{E}^{\prime} g\left(t, \overline{\widetilde{\xi}}_{t}\right), \\
& \widehat{F}\left(t, \bar{\xi}_{t}\right)=\mathbb{E}^{\prime} F\left(t, \bar{\xi}_{t}\right)-\mathbb{E}^{\prime} F\left(t, \overline{\widetilde{\xi}}_{t}\right), \\
& \widehat{G}\left(t, \bar{\xi}_{t}\right)=\mathbb{E}^{\prime} G\left(t, \bar{\xi}_{t}\right)-\mathbb{E}^{\prime} G\left(t, \overline{\widetilde{\xi}}_{t}\right), \\
& \widehat{\Phi}\left(\bar{y}_{T}\right)=\mathbb{E}^{\prime} \Phi\left(\bar{y}_{T}^{\prime}, \bar{y}_{T}\right)-\mathbb{E}^{\prime} \Phi\left(\overline{\tilde{y}}_{T}^{\prime}, \overline{\widetilde{y}}_{T}\right), \\
& \widehat{\Phi}\left(y_{T}\right)=\mathbb{E}^{\prime} \Phi\left(y_{T}^{\prime}, y_{T}\right)-\mathbb{E}^{\prime} \Phi\left(\tilde{y}_{T}^{\prime} \widetilde{y}_{T}\right) .
\end{aligned}
$$

By virtue of (H1)-(H3), we easily deduce

$$
\begin{aligned}
& \left(1-\alpha_{0}+\alpha_{0} \beta\right) \mathbb{E}\left|\hat{y}_{T}\right|^{2}+\mu \mathbb{E} \int_{0}^{T}\left(\left|\hat{y}_{t}\right|^{2}+\left|\widehat{z}_{t}\right|^{2}\right) d t \\
& \quad \leq \delta C \mathbb{E} \int_{0}^{T}\left(\left|\widehat{U}_{t}\right|^{2}+\left|\widehat{\bar{U}}_{t}\right|^{2}\right) d t+\delta C\left(\mathbb{E}\left|\hat{y}_{T}\right|^{2}+\mathbb{E}\left|\hat{\bar{y}}_{T}\right|^{2}\right),
\end{aligned}
$$


with some constant $C>0$. Hereafter, $C$ will be some generic constant, which can be different from line to line and depends only on the Lipschitz constants $k, \mu, \lambda$, and $\beta$. It is obvious that $1-\alpha_{0}+\alpha_{0} \beta \geq \bar{\beta}, \bar{\beta}=\min (1, \beta)>0$.

On the other hand, for the difference of the solutions $(\widehat{Y}, \widehat{Z})=(Y-\widetilde{Y}, Z-\widetilde{Z})$, we apply a standard method of estimation. Applying Itôs formula to $\left|\widehat{Y}_{t}\right|^{2}$ on $[t, T]$, we have

$$
\begin{aligned}
& \mathbb{E}\left|\widehat{Y}_{t}\right|^{2}+\mathbb{E} \int_{t}^{T}\left|\widehat{Z}_{s}\right|^{2} d s \\
& =\mathbb{E}\left|\alpha_{0} \widehat{\Phi}\left(y_{T}\right)+\left(1-\alpha_{0}\right) \widehat{y}_{T}+\delta\left(\widehat{\Phi}\left(\bar{y}_{T}\right)-\hat{\bar{y}}_{T}\right)\right|^{2} \\
& -2 \mathbb{E} \int_{t}^{T}\left\langle\widehat{Y}_{s}, \alpha_{0} \widehat{F}\left(s, \xi_{s}\right)-\left(1-\alpha_{0}\right) \mu \widehat{y}_{s}\right. \\
& \left.\quad+\delta\left(\widehat{F}\left(s, \bar{\xi}_{s}\right)+\mu \hat{\bar{y}}_{s}\right)\right\rangle d s \\
& +\mathbb{E} \int_{t}^{T}\left|\alpha_{0} \widehat{G}\left(s, \xi_{s}\right)-\left(1-\alpha_{0}\right) \mu \widehat{z}_{s}+\delta\left(\widehat{G}\left(s, \bar{\xi}_{s}\right)+\mu \hat{\bar{z}}_{s}\right)\right|^{2} d s,
\end{aligned}
$$

where

$$
\begin{gathered}
\widehat{F}\left(t, \xi_{t}\right)=\mathbb{E}^{\prime} F\left(t, \xi_{t}\right)-\mathbb{E}^{\prime} F\left(t, \tilde{\xi}_{t}\right), \\
\widehat{F}\left(t, \bar{\xi}_{t}\right)=\mathbb{E}^{\prime} F\left(t, \bar{\xi}_{t}\right)-\mathbb{E}^{\prime} F\left(t, \overline{\tilde{\xi}}_{t}\right), \\
\widehat{G}\left(t, \xi_{t}\right)=\mathbb{E}^{\prime} G\left(t, \xi_{t}\right)-\mathbb{E}^{\prime} G\left(t, \tilde{\xi}_{t}\right), \\
\widehat{G}\left(t, \bar{\xi}_{t}\right)=\mathbb{E}^{\prime} G\left(t, \bar{\xi}_{t}\right)-\mathbb{E}^{\prime} G\left(t, \overline{\tilde{\xi}}_{t}\right), \\
\widehat{\Phi}\left(y_{T}\right)=\mathbb{E}^{\prime} \Phi\left(y_{T}^{\prime}, y_{T}\right)-\mathbb{E}^{\prime} \Phi\left(\tilde{y}_{T}^{\prime} \tilde{y}_{T}\right), \\
\widehat{\Phi}\left(\bar{y}_{T}\right)=\mathbb{E}^{\prime} \Phi\left(\bar{y}_{T}^{\prime}, \bar{y}_{T}\right)-\mathbb{E}^{\prime} \Phi\left(\overline{\tilde{y}}_{T}^{\prime}, \overline{\tilde{y}}_{T}\right) .
\end{gathered}
$$

By virtue of (H2), we have

$$
\begin{aligned}
& \mathbb{E}\left|\widehat{Y}_{t}\right|^{2}+\mathbb{E} \int_{t}^{T}\left|\widehat{Z}_{s}\right|^{2} d s \\
& \leq 4 \mathbb{E}\left[\alpha_{0}^{2}\left|\widehat{\Phi}\left(y_{T}\right)\right|^{2}+\left(1-\alpha_{0}\right)^{2}\left|\widehat{y}_{T}\right|^{2}\right. \\
& \left.+\delta^{2}\left|\widehat{\Phi}\left(\bar{y}_{T}\right)\right|^{2}+\delta^{2}\left|\hat{\bar{y}}_{T}\right|^{2}\right] \\
& +2 \mathbb{E} \int_{t}^{T}|\widehat{Y} s|\left(\alpha_{0}\left|\widehat{F}\left(s, \xi_{s}\right)\right|+\left(1-\alpha_{0}\right) \mu\left|\hat{y}_{s}\right|\right. \\
& \left.+\delta\left|\widehat{F}\left(s, \bar{\xi}_{s}\right)\right|+\delta \mu\left|\hat{\bar{y}}_{s}\right|\right) d s \\
& +\mathbb{E} \int_{t}^{T}\left[\frac{1+2 \lambda}{4 \lambda} \alpha_{0}^{2}\left|\widehat{G}\left(s, \xi_{s}\right)\right|^{2}\right] d s \\
& +3 \mathbb{E} \int_{t}^{T}\left[\frac { 1 + 2 \lambda } { 1 - 2 \lambda } \left(\left(1-\alpha_{0}\right)^{2} \mu^{2}\left|\widehat{z}_{s}\right|^{2}\right.\right. \\
& \left.\left.\quad+\delta^{2}\left|\widehat{G}\left(s, \bar{\xi}_{s}\right)\right|^{2}+\delta^{2} \mu^{2}\left|\hat{\bar{z}}_{s}\right|^{2}\right)\right] d s
\end{aligned}
$$

$$
\begin{aligned}
& \leq C \mathbb{E}\left|\hat{y}_{T}\right|^{2}+\delta C \mathbb{E}\left|\hat{\bar{y}}_{T}\right|^{2} \\
& +\mathbb{E} \int_{t}^{T}\left[\left(\frac{8 k}{1-2 \lambda}\left|\widehat{Y}_{s}\right|^{2}+\frac{1-2 \lambda}{8 k}\left|\widehat{F}\left(s, \xi_{s}\right)\right|^{2}\right)\right. \\
& \left.+\left(1-\alpha_{0}\right) \mu\left(\left|\widehat{Y}_{s}\right|^{2}+\left|\widehat{y}_{s}\right|^{2}\right)\right] d s \\
& +\delta \mathbb{E} \int_{t}^{T}\left[\left(\left|\widehat{Y}_{s}\right|^{2}+\left|\widehat{F}\left(s, \bar{\xi}_{s}\right)\right|^{2}\right)+\mu\left(\left|\widehat{Y}_{s}\right|^{2}+\left|\hat{\bar{y}}_{s}\right|^{2}\right)\right] d s \\
& +\mathbb{E} \int_{t}^{T}\left[\frac{1+2 \lambda}{4 \lambda}\left|\widehat{G}\left(s, \xi_{s}\right)\right|^{2}\right] d s \\
& +3 \mathbb{E} \int_{t}^{T}\left[\frac { 1 + 2 \lambda } { 1 - 2 \lambda } \left(\left(1-\alpha_{0}\right)^{2} \mu^{2}\left|\widehat{z}_{s}\right|^{2}\right.\right. \\
& \left.\quad+\delta^{2} \mid \widehat{G}_{T}\left(s,\left.\bar{\xi}_{s}\right|^{2}+\delta^{2} \mu^{2}\left|\overline{\bar{z}}_{s}\right|^{2}\right)\right] d s \\
& +C \mathbb{E} \int_{t}^{T}\left|\widehat{Y}_{s}\right|^{2} d s+\left.\delta C \mathbb{E} \int_{t}^{T}\left|\hat{\bar{y}}_{T}\right|^{2}\right|^{2} d s \\
& +C \mathbb{E} \int_{t}^{T}\left(\left|\hat{y}_{s}\right|^{2}+\left|\widehat{z}_{s}\right|^{2}\right) d s+\frac{3+2 \lambda}{4} \mathbb{E} \int_{t}^{T}\left|\widehat{Z}_{s}\right|^{2} d s .
\end{aligned}
$$

Thus, we have

$$
\begin{aligned}
\mathbb{E}\left|\widehat{Y}_{t}\right|^{2} & +\frac{1-2 \lambda}{4} \mathbb{E} \int_{t}^{T}\left|\widehat{Z}_{s}\right|^{2} d s \\
\leq & C \mathbb{E} \int_{t}^{T}\left|\widehat{Y}_{s}\right|^{2} d s+C\left(\mathbb{E}\left|\hat{y}_{T}\right|^{2}+\delta \mathbb{E}\left|\hat{\bar{y}}_{T}\right|^{2}\right) \\
& +C \mathbb{E} \int_{t}^{T}\left(\left|\hat{y}_{s}\right|^{2}+\left|\widehat{z}_{s}\right|^{2}+\delta\left|\widehat{\bar{U}}_{s}\right|^{2}\right) d s .
\end{aligned}
$$

By Gronwall's inequality, it follows that

$$
\begin{aligned}
\mathbb{E}\left|\widehat{Y}_{t}\right|^{2}+\mathbb{E} \int_{t}^{T}\left|\widehat{Z}_{s}\right|^{2} d s \\
\leq C\left(\mathbb{E}\left|\hat{y}_{T}\right|^{2}+\delta \mathbb{E}\left|\hat{\bar{y}}_{T}\right|^{2}\right) \\
\quad+C \mathbb{E} \int_{0}^{T}\left(\left|\hat{y}_{t}\right|^{2}+\left|\widehat{z}_{t}\right|^{2}+\delta\left|\widehat{\bar{U}}_{t}\right|^{2}\right) d t .
\end{aligned}
$$

Then, we can deduce

$$
\begin{aligned}
& \mathbb{E} \int_{0}^{T}\left(\left|\widehat{Y}_{t}\right|^{2}+\left|\widehat{Z}_{t}\right|^{2}\right) d t \\
& \leq C\left(\mathbb{E}\left|\hat{y}_{T}\right|^{2}+\delta \mathbb{E}\left|\hat{\bar{y}}_{T}\right|^{2}\right) \\
& \quad+C \mathbb{E} \int_{0}^{T}\left(\left|\hat{y}_{t}\right|^{2}+\left|\widehat{z}_{t}\right|^{2}+\delta\left|\widehat{\bar{U}}_{t}\right|^{2}\right) d t .
\end{aligned}
$$


Combining the above two estimates (27) and (33), for a sufficiently large constant $C>0$, we easily have

$$
\begin{aligned}
& \mathbb{E} \int_{0}^{T}\left|\widehat{U}_{t}\right|^{2} d t+\mathbb{E}\left|\hat{y}_{T}\right|^{2} \\
& \quad \leq \delta C\left(\mathbb{E} \int_{0}^{T}\left|\widehat{U}_{t}\right|^{2} d t+\mathbb{E}\left|\hat{y}_{T}\right|^{2}+\mathbb{E} \int_{0}^{T}\left|\widehat{\bar{U}}_{t}\right|^{2} d t+\mathbb{E}\left|\widehat{\bar{y}}_{T}\right|^{2}\right) .
\end{aligned}
$$

We now choose $\delta_{0}=1 / 3 C$. It is clear that, for each fixed $\delta \in$ $\left[0, \delta_{0}\right]$, the mapping $I_{\alpha_{0}+\delta}$ is contractive in the sense that

$$
\mathbb{E} \int_{0}^{T}\left|\widehat{U}_{t}\right|^{2} d t+\mathbb{E}\left|\hat{y}_{T}\right|^{2} \leq \frac{1}{2}\left(\mathbb{E} \int_{0}^{T}\left|\widehat{\bar{U}}_{t}\right|^{2} d t+\mathbb{E}\left|\widehat{\bar{y}}_{T}\right|^{2}\right) .
$$

Thus, this mapping has a unique fixed point $U=(y, Y, z, Z) \epsilon$ $M^{2}\left(0, T ; \mathbb{R}^{n+n+n \times l+n \times d}\right)$, which is the solution of (21) for $\alpha=$ $\alpha_{0}+\delta$, as $\delta \in\left[0, \delta_{0}\right]$. The proof is complete.

Now we can obtain one of the main results in this paper which is the following existence and uniqueness theorem for solutions of MF-FBDSDE (17).

Theorem 3. Under assumptions (H1)-(H3), (17) has a unique solution in $M^{2}\left(0, T ; \mathbb{R}^{n+n+n \times l+n \times d}\right)$.

Proof. Uniqueness: let $U=(y, Y, z, Z)$ and $\bar{U}=(\bar{y}, \bar{Y}, \bar{z}, \bar{Z})$ be two solutions of (17). We use the same notations as in Lemma 2. Applying Itô's formula to $\langle\widehat{y}, \widehat{Y}\rangle$ on $[0, T]$, we have

$$
\mathbb{E}\left\langle\widehat{y}_{T}, \widehat{\Phi}\left(y_{T}\right)\right\rangle=\mathbb{E} \int_{0}^{T} \mathbb{E}^{\prime}\left\langle A\left(t, U_{t}\right)-A\left(t, \bar{U}_{t}\right), \widehat{U}_{t}\right\rangle d t
$$

By virtue of (H3), it follows that

$$
\mu \mathbb{E} \int_{0}^{T} \mathbb{E}^{\prime}\left\langle A\left(t, U_{t}\right)-A\left(t, \bar{U}_{t}\right), \widehat{U}_{t}\right\rangle d t \leq 0
$$

Thus, $U \equiv U^{\prime}$. The uniqueness is proven.

Existence: when $\alpha=0$, (21) has a unique solution in $M^{2}\left(0, T ; \mathbb{R}^{n+n+n \times l+n \times d}\right)$. It follows from Lemma 2 that there exists a positive constant $\delta_{0}=\delta_{0}(k, \lambda, \mu, \beta)$ such that, for any $\delta \in\left[0, \delta_{0}\right]$ and $x \in \mathbb{R}^{n}, \varphi \in L^{2}\left(\Omega, \mathscr{F}_{T}, P ; \mathbb{R}^{n}\right)$, and $\left(F_{0}, f_{0}, G_{0}, g_{0}\right) \in M^{2}\left(0, T ; \mathbb{R}^{n+n+n \times l+n \times d}\right),(21)$ has a unique solution for $\alpha=\delta$. Since $\delta_{0}$ depends only on $(k, \lambda, \mu, \beta)$, we can repeat this process for $N$ times with $1 \leq N \delta_{0}<1+\delta_{0}$. In particular, for $\alpha=1$ with $\left(F_{0}, f_{0}, G_{0}, g_{0}\right) \equiv 0$ and $\varphi \equiv 0$, (17) has a unique solution in $M^{2}\left(0, T ; \mathbb{R}^{n+n+n \times l+n \times d}\right)$. The proof is complete.
Remark 4. Condition (H3) can be replaced by the following condition.

$(\mathrm{H} 3)^{\prime}$

$$
\begin{array}{r}
\mathbb{E}^{\prime}\langle A(t, \xi)-A(t, \bar{\xi}), U-\bar{U}\rangle \geq \mu|U-\bar{U}|^{2}, \\
\forall U=(y, Y, z, Z)^{T}, \quad \bar{U}=(\bar{y}, \bar{Y}, \bar{z}, \bar{Z})^{T}, \\
U^{\prime}=\left(y^{\prime}, Y^{\prime}, z^{\prime}, Z^{\prime}\right)^{T}, \\
\bar{U}^{\prime}=\left(\bar{y}^{\prime}, \bar{Y}^{\prime}, \bar{z}^{\prime}, \bar{Z}^{\prime}\right)^{T} \in \mathbb{R}^{n} \times \mathbb{R}^{n} \times \mathbb{R}^{n \times l} \times \mathbb{R}^{n \times d}, \\
\forall t \in[0, T] \\
\mathbb{E}^{\prime}\left\langle\Phi\left(y, y^{\prime}\right)-\Phi\left(\bar{y}, \bar{y}^{\prime}\right), y-\bar{y}\right\rangle \leq-\beta|y-\bar{y}|^{2}, \\
\forall y, \quad \bar{y} \in \mathbb{R}^{n},
\end{array}
$$

where $\mu$ and $\beta$ are positive constants.

By similar arguments to Theorem 3, we have another parallel existence and uniqueness theorem for MF-FBDSDEs.

Theorem 5. Under assumptions (H1), (H2), and (H3)', MFFBDSDE (17) has a unique solution in $M^{2}\left(0, T ; R^{n+n+n \times l+n \times d}\right)$.

\section{Probabilistic Interpretation for a Class of Nonlocal SPDEs}

The connection of BDSDEs and systems of second-order quasilinear SPDEs was observed by Pardoux and Peng [1]. This can be regarded as a stochastic version of the wellknown Feynman-Kac formula which gives a probabilistic interpretation for second-order SPDEs of parabolic types. Thereafter this subject has attracted many mathematicians; refer to Bally and Matoussi [2], Gomez et al. [3], $\mathrm{Hu}$ and Ren [4], Ren et al. [5]; see also Zhang and Zhao [6-8]. One distinctive character of this result is that the forward component of the MF-FBDSDE is coupled with the backward variable. This section can be viewed as a continuation of such a theme and will exploit the above theory of fully coupled MF-FBDSDE in order to provide a probabilistic formula for the solution of a quasilinear nonlocal SPDE combined with algebra equations.

For each $x \in \mathbb{R}^{n}$, consider the following MF-FBDSDE:

$$
\begin{aligned}
& d y_{s}=\mathbb{E}^{\prime} f\left(s, \xi_{s}\right) d s+\mathbb{E}^{\prime} g\left(s, \xi_{s}\right) \vec{d} W_{s}-z_{s} \overleftarrow{d} B_{s} \\
& d Y_{s}=\mathbb{E}^{\prime} F\left(s, \xi_{s}\right) d s+\mathbb{E}^{\prime} G\left(s, \xi_{s}\right) \overleftarrow{d} B_{s}+Z_{s} \vec{d} W_{s} \\
& y_{t}=x, \quad Y_{T}=\mathbb{E}^{\prime} \Phi\left(y_{T}^{\prime}, y_{T}\right)
\end{aligned}
$$


where

$$
\begin{aligned}
\xi_{s}= & \left(y_{s}, Y_{s}, z_{s}, Z_{s}, y_{s}^{\prime}, Y_{s}^{\prime}, z_{s}^{\prime}, Z_{s}^{\prime}\right), \\
F:[ & t, T] \times \mathbb{R}^{n} \times \mathbb{R}^{n} \times \mathbb{R}^{n \times l} \times \mathbb{R}^{n \times d} \times \mathbb{R}^{n} \\
& \times \mathbb{R}^{n} \times \mathbb{R}^{n \times l} \times \mathbb{R}^{n \times d} \longrightarrow \mathbb{R}^{n}, \\
f:[ & t, T] \times \mathbb{R}^{n} \times \mathbb{R}^{n} \times \mathbb{R}^{n \times l} \times \mathbb{R}^{n \times d} \times \mathbb{R}^{n} \\
& \times \mathbb{R}^{n} \times \mathbb{R}^{n \times l} \times \mathbb{R}^{n \times d} \longrightarrow \mathbb{R}^{n}, \\
G: & {[t, T] \times \mathbb{R}^{n} \times \mathbb{R}^{n} \times \mathbb{R}^{n \times l} \times \mathbb{R}^{n \times d} \times \mathbb{R}^{n} } \\
& \times \mathbb{R}^{n} \times \mathbb{R}^{n \times l} \times \mathbb{R}^{n \times d} \longrightarrow \mathbb{R}^{n \times l}, \\
g: & {[t, T] \times \mathbb{R}^{n} \times \mathbb{R}^{n} \times \mathbb{R}^{n \times l} \times \mathbb{R}^{n \times d} \times \mathbb{R}^{n} } \\
& \times \mathbb{R}^{n} \times \mathbb{R}^{n \times l} \times \mathbb{R}^{n \times d} \longrightarrow \mathbb{R}^{n \times d}, \\
\Phi: & \mathbb{R}^{n} \times \mathbb{R}^{n} \longrightarrow \mathbb{R}^{n} .
\end{aligned}
$$

Assume that $(F, f, G, g, \Phi)$ in MF-FBDSDE (39) are deterministic, and MF-FBDSDE (39) has a unique measurable solution $\left(y_{s}, Y_{s}, z_{s}, Z_{s}\right), s \in[t, T]$. Set

$$
u(t, x):=Y_{t}^{t, x}, \quad v(t, x):=Z_{t}^{t, x}
$$

By the uniqueness of the solution to (39), it is known that, for any $t \leq s \leq T$,

$$
Y_{s}^{t, x}=Y_{s}^{s, y_{s}^{t, x}}=u\left(s, y_{s}^{t, x}\right)
$$

To simplify the notation, for $\varphi=F, f, G, g$, we define

$$
\begin{gathered}
\widehat{\varphi}\left(s, y_{s}^{0, x_{0}}, x\right) \\
\doteq \mathbb{E}\left[\varphi \left(s, y_{s}^{0, x_{0}}, x, u\left(s, y_{s}^{0, x_{0}}\right), u(s, x), \mu\left(s, y_{s}^{0, x_{0}}\right),\right.\right. \\
\left.\left.\mu(s, x), v\left(s, y_{s}^{0, x_{0}}\right), v(s, x)\right)\right] .
\end{gathered}
$$

According to our notations introduced in Section 2, we know that

$$
\begin{gathered}
\widehat{\varphi}\left(s, y_{s}^{0, x_{0}}, x\right) \\
=\mathbb{E}^{\prime}\left[\varphi \left(\omega^{\prime}, s, y^{0, x_{0}}\left(\omega^{\prime}, s\right), x, u\left(s, y^{0, x_{0}}\left(\omega^{\prime}, s\right)\right), u(s, x),\right.\right. \\
\mu\left(s, y^{0, x_{0}}\left(\omega^{\prime}, s\right)\right), \mu(s, x) \\
\left.\left.v\left(s, y^{0, x_{0}}\left(\omega^{\prime}, s\right)\right), v(s, x)\right)\right] .
\end{gathered}
$$

If there exists $u(t, x) \in C^{1,2}\left(\Omega \times[0, T] \times \mathbb{R}^{n} ; \mathbb{R}^{n}\right)$ solving the following quasilinear second-order nonlocal SPDE:

$$
\begin{aligned}
& u(t, x)=\mathbb{E}\left[\Phi\left(y_{T}^{0, x_{0}}, x\right)\right] \\
& +\int_{t}^{T}\left[\mathscr{L} u(s, x)+\widehat{F}\left(s, y_{s}^{0, x_{0}}, x\right)\right] d s \\
& +\int_{t}^{T} p \widehat{G}\left(s, y_{s}^{0, x_{0}}, x\right) \overleftarrow{d} B_{s} \\
& \nabla u(t, x) \mu(t, x)=q \widehat{G}\left(t, y_{t}^{0, x_{0}}, x\right), \quad p+q=1, \\
& q \neq 0, \quad p, q \in \mathbb{R}, \\
& v(t, x)=\nabla u(t, x) \hat{g}\left(t, y_{t}^{0, x_{0}}, x\right), \quad \forall(t, x) \in[0, T] \times \mathbb{R}^{n},
\end{aligned}
$$

where $u: \mathbb{R}_{+} \times \mathbb{R}^{n} \rightarrow \mathbb{R}^{m}$,

$$
\mathscr{L} u=\left(\begin{array}{c}
L u_{1} \\
\vdots \\
L u_{m}
\end{array}\right)
$$

with

$$
\begin{array}{rl}
L u_{k}(t, x):= & \sum_{i=1}^{n} \widehat{f}_{i}\left(t, y_{t}^{0, x_{0}}, x\right) \frac{\partial u_{k}}{\partial x_{i}}(t, x) \\
& +\frac{1}{2} \sum_{i, j=1}^{n} \mathbb{E}\left(\widehat{g} \widehat{g}^{T}\right)_{i j}\left(t, y_{t}^{0, x_{0}}, x\right) \frac{\partial^{2} u_{k}}{\partial x_{i} \partial x_{j}}(t, x) \\
& -\frac{1}{2} \sum_{i, j=1}^{n} \frac{\partial^{2} u_{k}}{\partial x_{i} \partial x_{j}}(t, x)\left(\mu(t, x) \mu(t, x)^{T}\right)_{i j} \\
k & k=1, \ldots, m,
\end{array}
$$

we can assert the following.

Theorem 6. Assume that (F, $f, G, g, \Phi)$ in MF-FBDSDE (39) are deterministic, and MF-FBDSDE (39) admits a unique measurable solution, the functions $F, f, G$, and $g$ are of class $C^{3}$. and $\Phi$ is of class $C^{2}$. If $(u, v)$ solves nonlocal SPDE (45), then (41) holds, where $(Y, Z)$ is determined uniquely by (39).

Proof. It suffices to show that $\left\{u\left(s, y_{s}^{t, x}\right), \widehat{g}\left(s, y_{s}^{0, x_{0}}, x\right)\right.$ $\left.\nabla u\left(s, y_{s}^{t, x}\right) ; 0 \leq s \leq t\right\}$ solves MF-FBDSDE (39).

Let $t=t_{0}<t_{1}<t_{2}<\cdots<t_{n}=T$; we have

$$
\begin{aligned}
& u\left(t_{i}, y_{t_{i}}^{t, x}\right)-u\left(t_{i+1}, y_{t_{i+1}}^{t, x}\right) \\
& \quad=u\left(t_{i}, y_{t_{i}}^{t, x}\right)-u\left(t_{i}, y_{t_{i+1}}^{t, x}\right)+u\left(t_{i}, y_{t_{i+1}}^{t, x}\right)-u\left(t_{i+1}, y_{t_{i+1}}^{t, x}\right)
\end{aligned}
$$




$$
\begin{aligned}
= & -\int_{t_{i}}^{t_{i+1}} \mathscr{L} u\left(t_{i}, y_{s}^{t, x}\right) d s+\int_{t_{i}}^{t_{i+1}} \nabla u\left(t_{i}, y_{s}^{t, x}\right) z_{s} \overleftarrow{d} B_{s} \\
& +\int_{t_{i}}^{t_{i+1}} \widehat{g}\left(t_{i}, y_{s}^{0, x_{0}}, y_{s}^{t, x}\right) \nabla u\left(s, y_{s}^{t, x}\right) \vec{d} W_{s} \\
& +\int_{t_{i}}^{t_{i+1}}\left[\mathscr{L} u\left(s, y_{t_{i+1}}^{t, x}\right)+\widehat{F}\left(s, y_{s}^{0, x_{0}}, y_{t_{i+1}}^{t, x}\right)\right] d s \\
& +\int_{t_{i}}^{t_{i+1}} p \widehat{G}\left(s, y_{s}^{0, x_{0}}, y_{t_{i+1}}^{t, x}\right) \overleftarrow{d} B_{s},
\end{aligned}
$$

where we have used Itô's formula and the equation satisfied by $u$. Finally, let the mesh size go to zero; we have

$$
\begin{aligned}
& u\left(t, y_{t}\right)-u\left(T, y_{T}\right) \\
& =\int_{t}^{T} \widehat{F}\left(s, y_{s}^{0, x_{0}}, y_{s}^{t, x}\right) d s+\int_{t}^{T} \widehat{G}\left(s, y_{s}^{0, x_{0}}, y_{s}^{t, x}\right) \overleftarrow{d} B_{s} \\
& \quad+\int_{t}^{T} \widehat{g}\left(s, y_{s}^{0, x_{0}}, y_{s}^{t, x}\right) \nabla u\left(s, y_{s}^{t, x}\right) \vec{d} W_{s} .
\end{aligned}
$$

It is easy to check that $Y_{s}^{t, x}:=u\left(s, y_{s}^{t, x}\right), Z_{s}^{t, x}:=\widehat{g}\left(s, y_{s}^{0, x_{0}}\right.$, $x) \nabla u\left(s, y_{s}^{t, x}\right)$ coincides with the unique solution to MFBDSDE of (39).

\section{Remark 7.}

(i) In the case when $p=0$ in nonlocal SPDE (45), nonlocal SPDE (45) will degenerate to the following nonlocal PDE:

$$
\begin{aligned}
& u(t, x)=\mathbb{E}\left[\Phi\left(y_{T}^{0, x_{0}}, x\right)\right] \\
& +\int_{t}^{T}\left[\mathscr{L} u(s, x)+\widehat{F}\left(s, y_{s}^{0, x_{0}}, x\right)\right] d s, \\
& \nabla u(t, x) \mu(t, x)=\widehat{G}\left(t, y_{t}^{0, x_{0}}, x\right) \text {, } \\
& v(t, x)=\nabla u(t, x) \hat{g}\left(t, y_{t}^{0, x_{0}}, x\right), \quad \forall(t, x) \in[0, T] \times \mathbb{R}^{n} .
\end{aligned}
$$

(ii) Equation (41) can be called a Feynman-Kac formula for nonlocal SPDE (45).

(iii) Equation (41) generalizes the PDE combined with algebra equations in $[32,33]$ to the mean-field case.

(iv) By virtue of a connection between them and fully coupled FBDSDE of mean-field type, Theorem 6 gives a probabilistic interpretation for the solutions to such kind of SPDE (45). Furthermore, the uniqueness for SPDE (45) is an interesting problem, and we hope to be able to address this issue in our future publications.

\section{Conflict of Interests}

The authors declare that there is no conflict of interests regarding the publication of this paper.

\section{Acknowledgments}

The authors would like to thank the anonymous referees and the editors for their helpful comments and suggestions. This work was supported by the National Natural Science Foundation of China (Grant nos. 11371226, 11071145, 11301298, 11201268, and 11231005), Foundation for Innovative Research Groups of National Natural Science Foundation of China (Grant no. 11221061), the 111 Project (Grant no. B12023), and the Natural Science Foundation of Shandong Province of China (Grant no. ZR2012AQ013).

\section{References}

[1] É. Pardoux and S. G. Peng, "Backward doubly stochastic differential equations and systems of quasilinear SPDEs," Probability Theory and Related Fields, vol. 98, no. 2, pp. 209-227, 1994.

[2] V. Bally and A. Matoussi, "Weak solutions for SPDEs and backward doubly stochastic differential equations," Journal of Theoretical Probability, vol. 14, no. 1, pp. 125-164, 2001.

[3] A. Gomez, K. Lee, C. Mueller, A. Wei, and J. Xiong, "Strong uniqueness for an SPDE via backward doubly stochastic differential equations," Statistics \& Probability Letters, vol. 83, no. 10, pp. 2186-2190, 2013.

[4] L. Y. Hu and Y. Ren, "Stochastic PDIEs with nonlinear Neumann boundary conditions and generalized backward doubly stochastic differential equations driven by Lévy processes," Journal of Computational and Applied Mathematics, vol. 229, no. 1, pp. 230-239, 2009.

[5] Y. Ren, A. Lin, and L. Y. Hu, "Stochastic PDIEs and backward doubly stochastic differential equations driven by Lévy processes," Journal of Computational and Applied Mathematics, vol. 223, no. 2, pp. 901-907, 2009.

[6] Q. Zhang and H. Z. Zhao, "Stationary solutions of SPDEs and infinite horizon BDSDEs," Journal of Functional Analysis, vol. 252, no. 1, pp. 171-219, 2007.

[7] Q. Zhang and H. Z. Zhao, "Stationary solutions of SPDEs and infinite horizon BDSDEs with non-Lipschitz coefficients," Journal of Differential Equations, vol. 248, no. 5, pp. 953-991, 2010.

[8] Q. Zhang and H. Z. Zhao, "SPDEs with polynomial growth coefficients and the Malliavin calculus method," Stochastic Processes and Their Applications, vol. 123, no. 6, pp. 2228-2271, 2013.

[9] S. G. Peng and Y. F. Shi, "A type of time-symmetric forwardbackward stochastic differential equations," Comptes Rendus Mathematique, vol. 336, no. 9, pp. 773-778, 2003.

[10] Q. F. Zhu, Y. F. Shi, and X. J. Gong, "Solutions to general forward-backward doubly stochastic differential equations," Applied Mathematics and Mechanics, vol. 30, no. 4, pp. 517-526, 2009.

[11] Q. F. Zhu and Y. F. Shi, "Forward-backward doubly stochastic differential equations and related stochastic partial differential equations," Science China Mathematics, vol. 55, no. 12, pp. 25172534, 2012.

[12] Y. C. Han, S. G. Peng, and Z. Wu, "Maximum principle for backward doubly stochastic control systems with applications," SIAM Journal on Control and Optimization, vol. 48, no. 7, pp. 4224-4241, 2010.

[13] L. Q. Zhang and Y. F. Shi, "Maximum principle for forwardbackward doubly stochastic control systems and applications," 
ESIAM: Control, Optimisation and Calculus of Variations, vol. 17, no. 4, pp. 1174-1197, 2011.

[14] Y. F. Shi and Q. F. Zhu, "Partially observed optimal controls of forward-backward doubly stochastic systems," ESIAM: Control, Optimisation and Calculus of Variations, vol. 19, no. 3, pp. 828843, 2013.

[15] M. Kac, "Foundations of kinetic theory," in Proceedings of the $3 r d$ Berkeley Symposium on Mathematical Statistics and Probability, vol. 3, pp. 171-197, Berkeley, Calif, USA, 1956.

[16] H. P. McKean, Jr., "A class of Markov processes associated with nonlinear parabolic equations," Proceedings of the National Academy of Sciences of the United States of America, vol. 56, no. 6, pp. 1907-1911, 1966.

[17] N. Ahmed, "Nonlinear diffusion governed by McKean-Vlasov equation on Hilbert space and optimal control," SIAM Journal on Control and Optimization, vol. 46, no. 1, pp. 356-378, 2007.

[18] N. Ahmed and X. Ding, "A semilinear McKean-Vlasov stochastic evolution equation in Hilbert space," Stochastic Processes and Their Applications, vol. 60, no. 1, pp. 65-85, 1995.

[19] V. Borkar and K. Kumar, "McKean-Vlasov limit in portfolio optimization," Stochastic Analysis and Applications, vol. 28, no. 5, pp. 884-906, 2010.

[20] T. Chan, "Dynamics of the McKean-Vlasov equation," The Annals of Probability, vol. 22, no. 1, pp. 431-441, 1994.

[21] D. Crisan and J. Xiong, "Approximate McKean-Vlasov representations for a class of SPDEs," Stochastics, vol. 82, no. 1-3, pp. 5368, 2010.

[22] P. Kotelenez, "A class of quasilinear stochastic partial differential equations of McKean-Vlasov type with mass conservation," Probability Theory and Related Fields, vol. 102, no. 2, pp. 159188, 1995.

[23] P. Kotelenez and T. Kurtz, "Macroscopic limits for stochastic partial differential equations of McKean-Vlasov type," Probability Theory and Related Fields, vol. 146, no. 1-2, pp. 189-222, 2010.

[24] J. Lasry and P. Lions, "Mean field games," Japanese Journal of Mathematics, vol. 2, no. 1, pp. 229-260, 2007.

[25] D. Dawson, "Critical dynamics and fluctuations for a mean-field model of cooperative behavior," Journal of Statistical Physics, vol. 31, no. 1, pp. 29-85, 1983.

[26] M. Y. Huang, R. Malhamé, and P. Caines, "Large population stochastic dynamic games: closed-loop McKean-Vlasov systems and the Nash certainty equivalence principle," Communications in Information and Systems, vol. 6, no. 3, pp. 221-252, 2006.

[27] R. Buckdahn, B. Djehiche, J. Li, and S. Peng, "Mean-field backward stochastic differential equations: a limit approach," The Annals of Probability, vol. 37, no. 4, pp. 1524-1565, 2009.

[28] T. X. Wang, Y. F. Shi, and Q. F. Zhu, "Mean-field backward doubly stochastic differential equations and applications," http://arxiv.org/abs/1108.5590.

[29] H. Du, Y. Peng, and Y. Wang, "Mean-field backward doubly stochastic differential equations and its applications," in Proceedings of the 31st Chinese Control Conference (CCC '12), pp. 1547-1552, Hefei, China, 2012.

[30] R. M. Xu, "Mean-field backward doubly stochastic differential equations and related SPDEs," Boundary Value Problems, vol. 2012, article 114, 2012.

[31] R. Buckdahn, J. Li, and S. G. Peng, "Mean-field backward stochastic differential equations and related partial differential equations," Stochastic Processes and Their Applications, vol. 119, no. 10, pp. 3133-3154, 2009.
[32] Z. Wu and Z. Y. Yu, "Fully coupled forward-backward stochastic differential equations and related partial differential equations system," Chinese Annals of Mathematics A, vol. 25, no. 4, pp. 457-468, 2004.

[33] Z. Wu and Z. Y. Yu, "Probabilistic interpretation for systems of parabolic partial differential equations combined with algebra equations".

[34] J. Li and Q. M. Wei, "Optimal control problems of fully coupled FBSDEs and viscosity solutions of Hamilton-Jacobi-Bellman equations," http://arxiv.org/abs/1302.0935.

[35] D. Nualart and É. Pardoux, "Stochastic calculus with anticipating integrands," Probability Theory and Related Fields, vol. 78, no. 4, pp. 535-581, 1988. 


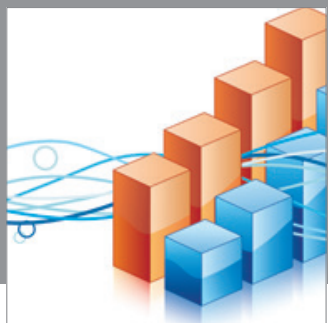

Advances in

Operations Research

mansans

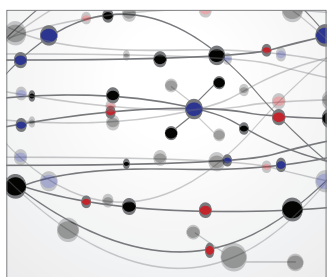

The Scientific World Journal
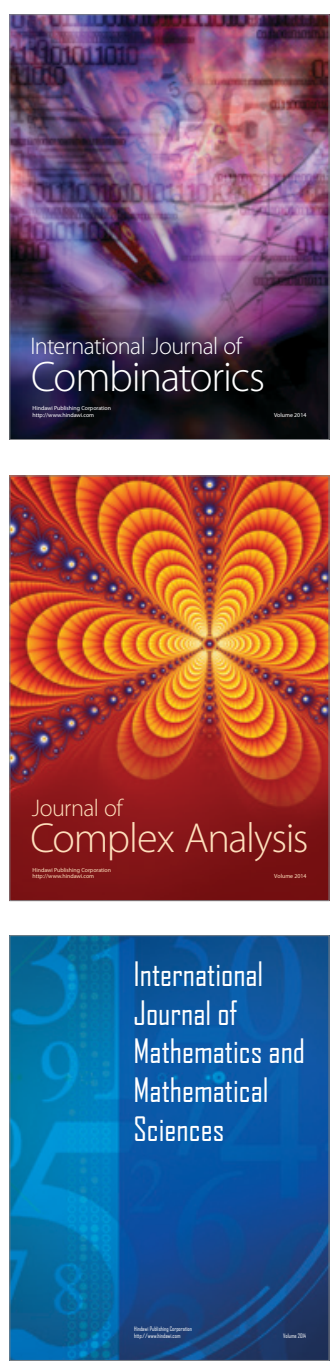
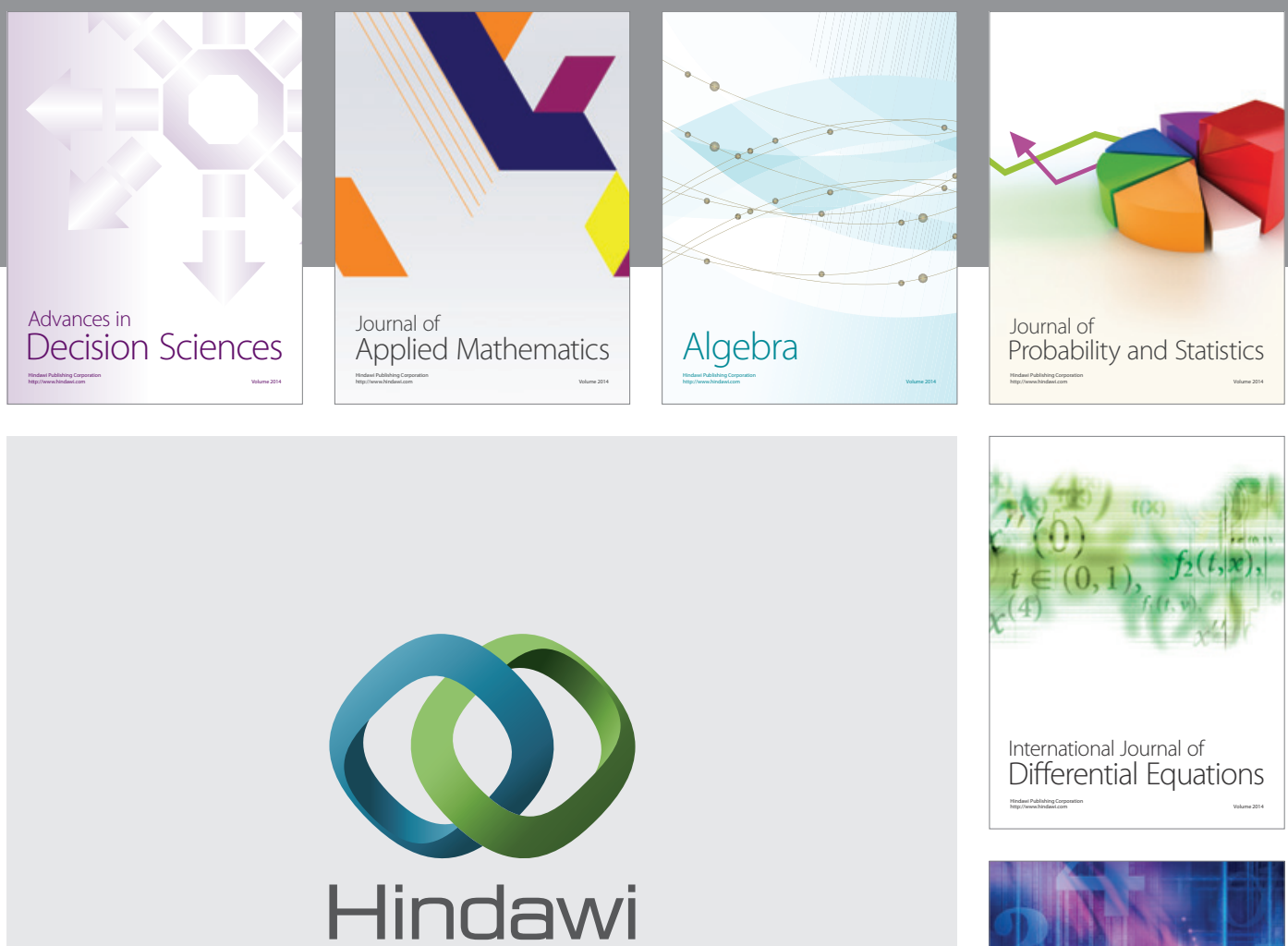

Submit your manuscripts at http://www.hindawi.com
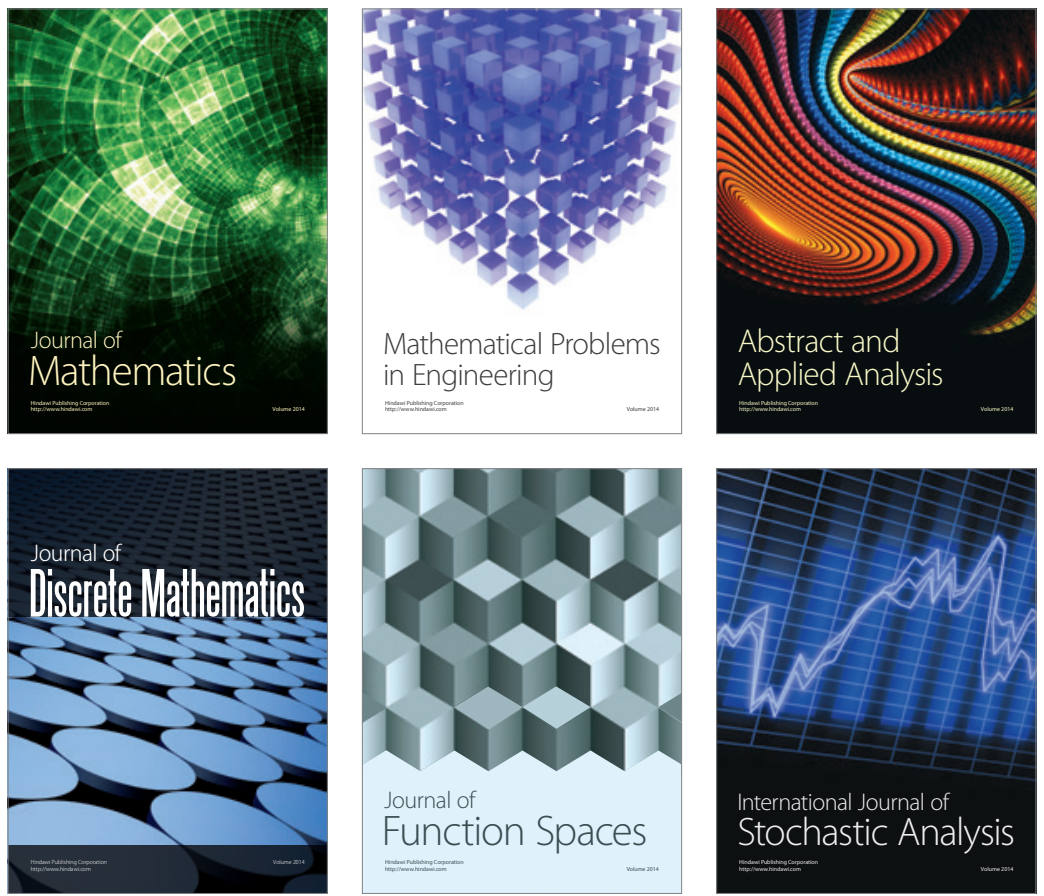

Journal of

Function Spaces

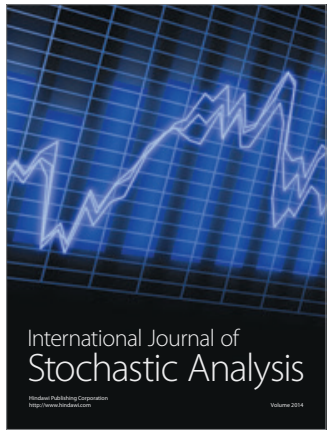

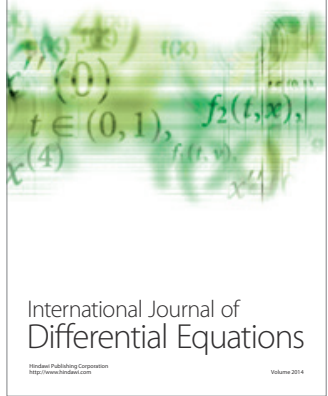
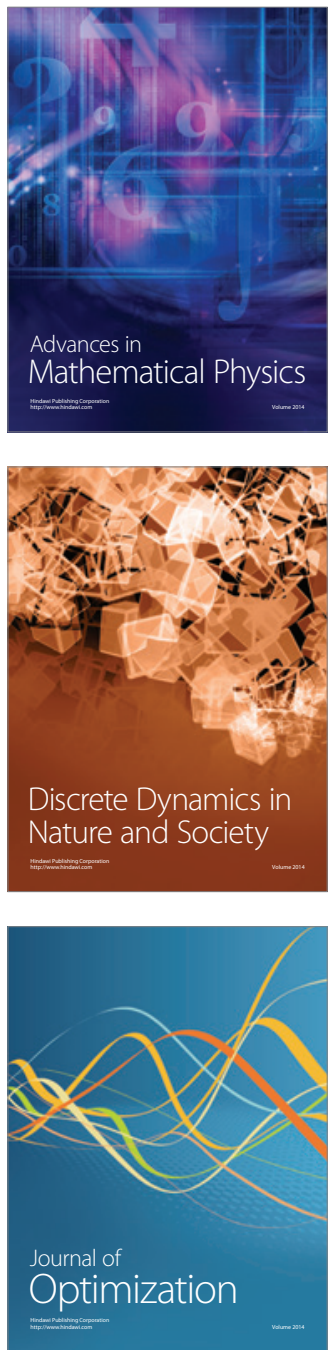